\title{
Oral mucositis in children with cancer and their relationship with limitation of activities
}

\author{
Miguel Ángel Palomo-Colli" ${ }^{*}$, Diana Carolina Soltero-Becerril², Luis Enrique Juárez Villegas ${ }^{1}$, \\ Carlo Cicero-Oneto ${ }^{1}$, José Félix Gaytán-Morales ${ }^{1}$, Iván Castorena-Villa ${ }^{1}$ and Mónica Mier-Cabrera ${ }^{3}$ \\ ${ }^{1}$ Department of Oncology; ${ }^{2}$ Department of Medical Pediatrics. Hospital Infantil de México Federico Gómez, Ciudad de México; ${ }^{3}$ Departamento of \\ Oncology, Hospital Regional de Alta especialidad de Ixtapaluca, Ixtapaluca. Mexico
}

\begin{abstract}
Introduction: Oral mucositis (OM) is a common chemo and radiotherapy adverse effect in oncological pediatric patients, oral complications may be observed during chemotherapy and are important side effects that may directly affect the anticancer treatment. Methods: We included 420 children and monitored them for 2 weeks after the initiation of chemotherapy between June 2012 and June 2016. Subjects were asked to use a daily diary to complete the related questions of the OM Daily Questionnaire from the start of chemotherapy to day 14. Results: Overall, $73 \%(n=307)$ of paediatric patients developed OM. Most cases were Grade 2 and 3. Of these, $12 \%(n=32)$ were Grade 1, and 49\% $(n=205)$ Grade 2. The mean time to onset of OM was 4.5 (standard deviation [SD]: 2.5; range: 2-9; $95 \%$ confidence interval [CI]: 3.8-5.3) days after the start of chemotherapy, with a mean duration of 6.5 (SD: 4; range: 2-13; 95\% Cl: 5.2-9.4) days and with the peak at day 7.1 (SD: 2.8; range: 4-11; 95\% Cl: 6.8-8.4). Conclusions: $O M$ is associated with negative effects on clinical outcomes.
\end{abstract}

Key words: Mucositis. Cancer. Chemotherapy. Quality of life, Children.

Correspondence:

*Miguel Ángel Palomo-Colli E-mail: phalomi@gmail.com 1665-9201/@ 2018 Sociedad Mexicana de Oncología. Published by Permanyer México. This is an Open Access article under the terms of the CC BY-NC-ND license (http://creativecommons.org/licenses/by-nc-nd/4.0/).

Available online: $30-05-2019$ Gac Mex Oncol. 2018;17:36-41 www.gamo-smeo.com
ms of the CC BY-NC-ND 


\section{Introduction}

Oral mucositis (OM) refers to the erythematous and ulcerative lesions of the oral mucosa observed in cancer patients treated with chemotherapy and/or radiotherapy in areas involving the oral cavity. This term, as described by Köstler ${ }^{1}$, emerged in the late 1980s to define inflammation in the oral cavity induced by chemotherapy and radiotherapy, since this inflammation represented a distinct entity from other oral lesions, called stomatitis, and had different etiopathogenesis. However, according to other authors, mucositis is an inflammation of the mucous membranes that leads to ulceration and rupture of their integrity, and can affect the entire gastrointestinal tract, from the mouth to the anus ${ }^{2}$.

$\mathrm{OM}$ is the inflammation that takes place in the oral epithelium as a consequence of antineoplastic treatments such as radiotherapy, chemotherapy or hematopoietic stem cell transplantation, and that is rather common during the treatment of oncological-hematological conditions.

An estimate of up to $50 \%$ of patients receiving chemotherapy for solid tumors or lymphomas develop oral or gastrointestinal mucositis.

Mucositis is estimated to occur in $22 \%$ of cases in the oral mucosa, in $8 \%$ in the oral and gastrointestinal mucosa, and in $7 \%$ in the gastrointestinal mucosa of patients treated with chemotherapy. However, in patients receiving high-dose chemotherapy as part of regimens for hematopoietic stem cell transplantation, it can occur in up to $75-80 \%^{3,4}$.

Mucosal barrier lesion is a complication that many children with oncological diseases experience during their treatment. Mucosal barrier lesion can be quite painful for patients and put them at high risk of developing complications. When the mucosal barrier is ruptured, the patient is at risk of developing a serious infection that can be life threatening. Patients who receive chemotherapy often develop neutropenia, which implies a higher risk of infections if added to mucosal damage.

The ability to recognize patients at high risk of having mucosal barrier lesions can stop the progression of the lesion and allows early treatment to avoid further complications.

$\mathrm{OM}$ is a common secondary event that deteriorates patient quality of life, worsens clinical conditions, increases the risk of infection and can lead to a delay in chemotherapy administration schedules, which drives to suboptimal dose intensity, which can ultimately affect the prognosis of the child with cancer. $\mathrm{OM}$ is the
Table 1. WHO oral toxicity scale

\begin{tabular}{|l|l|}
\hline Grade & Symptoms \\
\hline 0 & No subjective or objective evidence of mucositis \\
\hline 1 & Oral soreness with or without erythema, no ulcers \\
\hline 2 & Erythema and ulceration, can eat solids \\
\hline 3 & Erythema and ulceration, liquid diet is required \\
\hline 4 & Erythema and ulceration, alimentation not possible \\
\hline
\end{tabular}

second cause of dose-limiting toxicity in patients receiving chemotherapy. In addition, it can be the cause of hospital admissions, prolong hospital length of stay and sometimes predispose to admission to intensive care units.

The incidence of oral complications can be reduced by carrying out assessments of the oral cavity prior to starting therapy, eliminating potential sources of infection or irritation and using preventive measures throughout the treatment.

The risk factors for developing mucositis are classically associated with antineoplastic drugs modality, intensity and administration route.

Combination therapy can increase the severity of mucositis.

Children on antineoplastic treatment have been shown that have a higher likelihood of mucositis due to the high rate of cell turnover, just as people older than 50 years of age; other additional factors include poorly maintained oral cavity, dental pathology and defective prostheses, which must be solved prior to the start of therapy. The degree and severity of mucositis in cancer patients are determined by the type of employed drug, the dose used and patient genetic susceptibility ${ }^{5-7}$.

\section{Oral mucositis assessment}

Over the past 30 years, different models have been developed to document and quantify changes in oral cavity epithelial tissues and alterations in their function during and after cancer treatment. Currently, there is a wide variety of scales to assess the extent and severity of $\mathrm{OM}$, which are useful in clinical practice and as a research resource?.

The World Health Organization (WHO) defines the degree of mucositis lesions according to their severity (Table 1). It is a scale that is simple and easy-to-use in clinical practice, which combines both objective and subjective parameters to assess the degree of oral mucosa injury. 


\section{Mucositis economic impact}

Patients suffering from a significant degree of $\mathrm{OM}$ require supportive measures, such as the use of total parenteral nutrition, fluid replacement and antibiotic prophylaxis. This can substantially elevate the cost of treatment.

In a study conducted at the University Hospital of Connecticut, estimated cost for hospitalization was USD \$ 3,893 per chemotherapy cycle without mucositis, USD \$ 6,277 per chemotherapy cycle with OM and USD $\$ 9,132$ per chemotherapy cycle with both oral and gastrointestinal mucositis ${ }^{8}$.

Each grade of mucositis severity increase was found to be associated with an additional day of fever, an increase of twice the risk of severe infection, 2.7 additional days of total parenteral nutrition, 2.6 more days of analgesic treatment, an increase of 2.6 days in hospital length of stay and an increase of 3.7 in the risk of mortality per 100 days of in-hospital stay, which together contributes to an additional cost of USD \$25,000 to hospitalization costs 9 .

\section{Material and methods}

This prospective, observational, cohort study was carried out from June 2012 to June 2016 at the Oncology Department of the Federico Gómez Children's Hospital of Mexico. Three-year-old or older children on chemotherapy treatment for any type of neoplasm, who were willing to participate in the study (as well as their primary caregivers) and who were able to read and answer the pediatric OM daily questionnaire (OMDQ) adapted final version ${ }^{10}$ were selected. This was carried out only in patients admitted to the short-stay chemotherapy area.

The WHO scale was used to establish OM and its grades (Table 1).

The application of the instrument (OMDQ) was explained by pediatricians and oncologists to caregivers and children during their stay at the chemotherapy short-stay rooms (hospitalization), with 10-15 minutes being required. The respondents answered the questionnaire for 14 days, starting 24 hours after the start of the chemotherapy scheme and the responses were recorded at the end of the 24 hours of the calendar date.

With regard to the OMDQ daily questionnaire, the presence of soreness in the mouth and throat was assessed every 24 hours using facial symbols incorporated to the child's self-completed version, which were adapted from the Wong-Baker FACES pain score scale.
The face scale has demonstrated reliability and validity in the use of patient self-reported pain in children ${ }^{11}$.

From the OM diary (OMDQ), four questions were separately evaluated: 1) pain intensity in the mouth and throat, 2) mucositis impact on eating, 3) on drinking and 4) on talking.

One doctor examined the children's oral cavity when they referred the presence of soreness in order to determine the state of the oral cavity and decide the type of treatment that was required. All the children who presented with mucositis were assessed and treated according to their evolution (as outpatients or inpatients).

\section{Results}

Total number of chemotherapies administered in the hospitalization area during the study period was 4,215 infusions and 420 children who agreed to participate were included in the study. According to gender, 240 were boys (57\%) and 180 girls (43\%). Administered drugs included cytosine arabinoside $(28 \%)$, high-dose methotrexate $\left(2 \mathrm{~g} / \mathrm{m}^{2}\right.$ or higher) $(35 \%)$, anthracyclines $(37 \%)$, etoposide $(14 \%)$, vincristine $(12 \%)$, cyclophosphamide (9\%) and others (13\%).

Using the WHO mucositis assessment scale, among the 420 patients included, $113(27 \%)$ were found not to have OM, 51 children (12\%) had grade 1 mucositis, 205 children (49\%) grade 2, $46(11 \%)$ grade 3 and five children (1\%) had grade 4 mucositis. The percentage of patients who experienced $\mathrm{OM}$ in the entire study group was $73 \%(n=307)$ (Fig. 1).

Mean time for the development of OM was 4.5 days (SD: 2.5; range: $2-9$ : $95 \% \mathrm{Cl}: 3.8-5.3)$ days after the start of chemotherapy, with a mean duration of 6.5 days (SD: 4; range: 2-13, 95\% Cl: 5.2-9.4) with a peak at day 7.1 (SD: 2.8; range: 4-11, 95\% Cl: 6.8-8.4).

The lesions were described according to the OM assessment scale (OMAS) ${ }^{7,9}$. Of the 307 patients with some degree of mucositis, $14 \%$ only had mucosal erythema, $51 \%$ had erythema and an ulcer of less than $1 \mathrm{~cm}, 23 \%$ had ulcerative lesions larger than $1 \mathrm{~cm}$, the remaining $10 \%$ had confluent ulcers larger than $3 \mathrm{~cm}$ and $2 \%$ had necrosis and bleeding from the lesions.

Among the patients with different degrees of mucositis $(n=307), 47 \%$ were found to refer tolerable pain, $38 \%$ moderate pain, $12 \%$ severe pain and $3 \%$ intolerable pain (Fig. 2).

Findings regarding food intake during the mucositis episodes were the following: $41 \%$ tolerated solid diet, $46 \%$ had difficulty swallowing solids and were offered liquid diet, and the remaining $13 \%$ did not tolerate any 


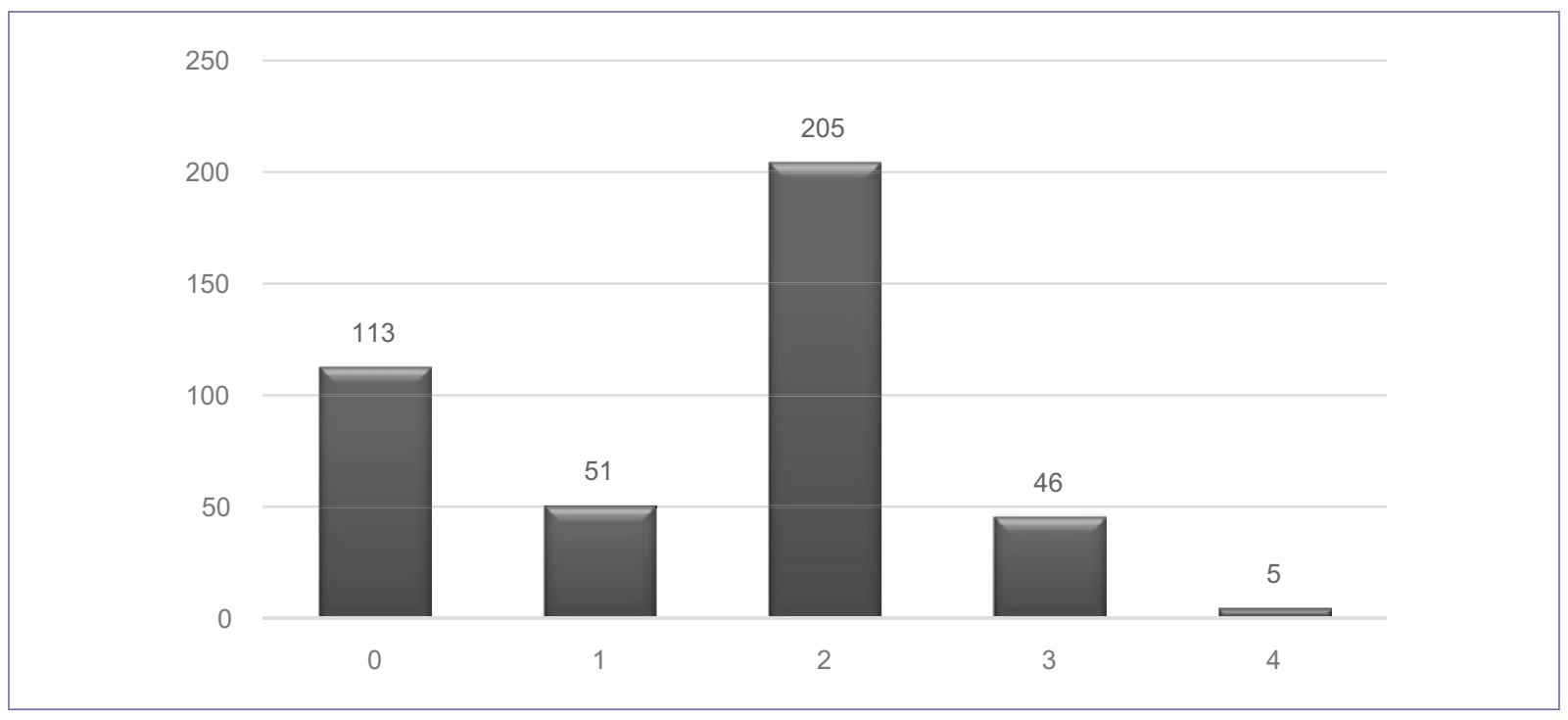

Figure 1. Mucositis grades according to WHO.

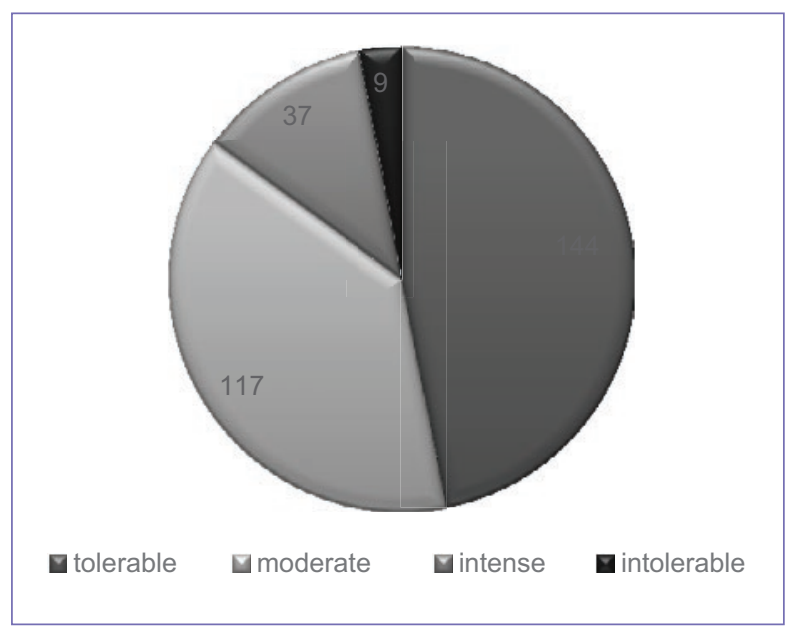

Figure 2. Pain in children with mucositis.

of both diets and had to remain on fasting until the remission of symptoms with intravenous fluids and/or parenteral nutrition, according to the time it took to control the pain (Fig. 3).

Among the 141 children who only tolerated fluids, $43 \%$ had no limitations to drink, $33 \%$ had slightly limited drinking capacity and $24 \%$ showed drinking limitation and presence of sialorrhea (Fig. 3).

Regarding the limitation to talk in children with mucositis $(n=307), 53 \%$ had no limitations, $24 \%$ referred some pain when talking, $13 \%$ had moderate pain when speaking, and $10 \%$ were unable to talk due to extreme pain (Fig. 3).
The treatment for $\mathrm{OM}$ in the institution where the study was carried out does not adhere to any clinical practice guidelines, since, as previously mentioned, these are rather diverse. Children with grade 1 and grade 2 mucositis with no pain or tolerable pain were offered normal or soft diet, mouth washing with carbonated water and mouthwashes with Gelclair ${ }^{\circledR}$ to protect the area with lesions and analgesics as required. In children with grade 2 or 3 mucositis with moderate or intense pain, soft or liquid diet was given, as well as rinses with cold fluids, rinses with Gelclair ${ }^{\circledR}$ and topical and/or systemic analgesia by oral route. Children with grade 3 or 4 mucositis and intolerable pain were treated with fasting, parenteral solutions and/or total parenteral nutrition as required, rinses with Gelclair ${ }^{\circledR}$ and intravenous analgesics as required.

Hospitalization was required by 40 children (13\%) due to the presence of intense and intolerable pain, grade 3 and 4 mucositis or due to association with neutropenia and fever. Mean hospitalization time was 6 days (SD: 2.5 ; range: $3-28 ; 95 \% \mathrm{Cl}$ : 3.8-12.3), with all hospitalized children requiring systemic antibiotics.

\section{Discussion}

$\mathrm{OM}$ is an adverse effect in children receiving treatment. In our study, the percentage of occurrence is high (73\%), with grades 3 and 4 occurring in 15 and 1.6\%, respectively. The elevated OM percentage is probably due to selection bias, since not all children who required chemotherapy in the assessed period agreed to 


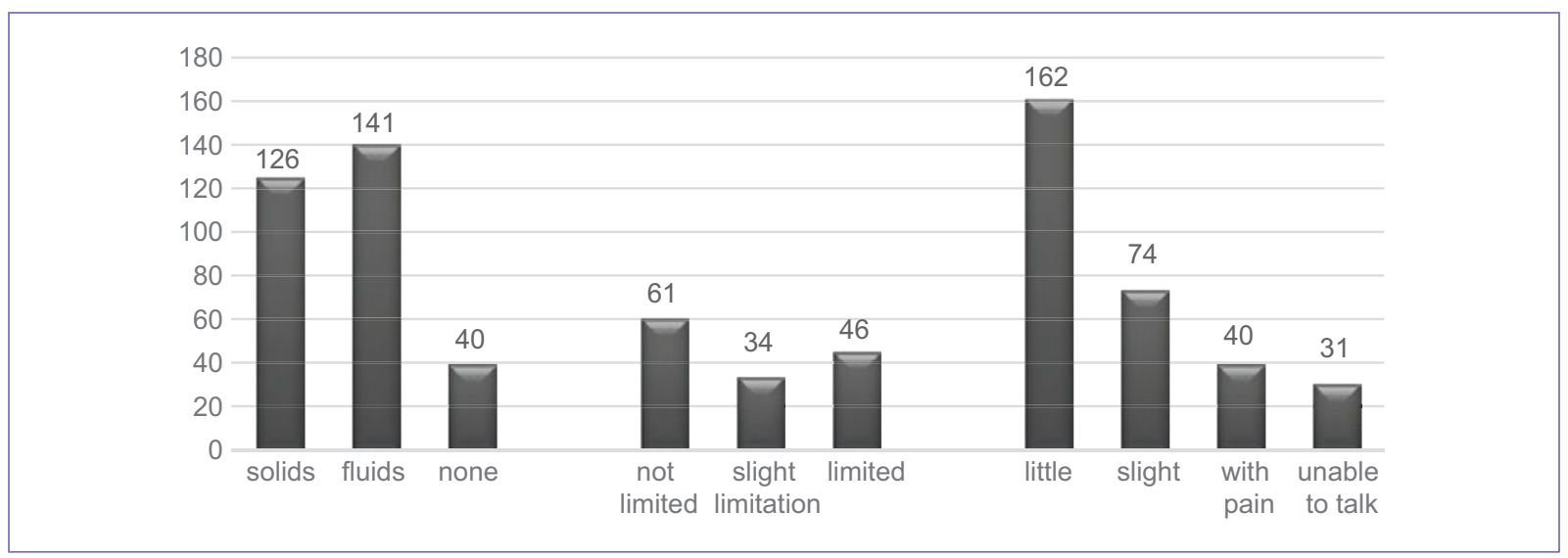

Figure 3. Limitations of activities in children with mucositis (swallowing, drinking and talking).

participate in the study, and there is the chance that children who had this complication were those who completed the evaluation.

OM-related pain in this study determines a considerable proportion of limitations related to the oral cavity function, reflected on the limitations for the intake of solids and fluids and to talk, in comparison with the study by Ip et al., where scores related to activity limitations in children are assessed (mouth and throat soreness [MTS] score), with a range of 18 to $35 \%$ being referred for activities such as eating, drinking, talking, swallowing and sleeping, and where $25 \%$ of the study population had limitations related to all evaluated activities and 39\% reported limitation in at least two activities. In the assessment carried out in our population, severe pain (intense and intolerable), which has an impact related to the basic activities of eating and drinking, occurred in $15 \%$ of the population; among these patients, $10 \%$ were unable to talk due to mucositis-associated pain and $25 \%$ had severe limitations for the activities of eating, drinking and talking. Unlike the referred study, we did not assess limitations for the sleeping and swallowing functions.

In those patients who have high scores in the assessment of mouth and throat soreness (MTS 3-4), there is a relationship with weight loss $\geq 2 \mathrm{~kg}$, as well as with higher use of intravenous fluid therapy, use of analgesics and oral and intravenous systemic antibiotics. In our study group, it was not possible to assess the impact of weight decrease, since previous weight registry and at the end of the resolution of the mucositis event was not systematically determined ${ }^{12}$.

It is important mentioning that the number of hospitalizations in studies related to mucositis is low or they are not required, in contrast with the assessed group, where hospitalization was required in $13 \%$ of children due to associated comorbidities. In this study, we did not assess factors associated with mucositis. All children with neutropenia were hospitalized and treated with intravenous antibiotics.

Children with neutropenia have an increased risk of severe mucositis owing to low neutrophil count, which are essential to protect the mucosa, and may also affect oral epithelial cells proliferation capacity ${ }^{13}$. In addition to increasing the risk of colonization, damaged oral mucosa allows an increase of pro-inflammatory cytokines, which favors mucositis worsening ${ }^{14}$. There are other factors, such as genetics, that can be predisposing to the development of mucositis and constitute a potential area of interest for further study.

\section{Conclusions}

We consider it necessary to monitor all children with cancer who require drugs that cause damage to the mucosa and provide early treatment in order to reduce the number of hospitalizations related to this complication. In addition, it is necessary to treat all the conditions that favor the problem (caries, poor oral hygiene, deficiency in oral hygiene techniques, use of drugs and substances that increase the risk). Quality of life assessment in children with cancer should be an essential part for comprehensive treatment and decision making in order to improve care and reduce the factors that can impact on non-adherence to treatment.

\section{Conflict of interests}

The authors declare not having any conflict of interest. 


\section{Acknowledgement}

Our special thanks to all the children and their families who gave their time to this study. We also express our gratitude to the students, doctors and nurses who participated in different aspects of this study.

\section{References}

1. Köstler WJ, Hejna M, Wenzel C, Zielinski CC. Oral mucositis complicating chemotherapy and/or radiotherapy: options for prevention and treatment. CA Cancer J Clin. 2001;51:290-315.

2. McGuire DB. Mucosal tissue injury in cancer therapy. More than muscositis and mouthwash. Cancer Pract. 2002;10:179-91.

3. Elting LS, Cooksley C, Chambers M, Cantor SB, Manzullo E, Rubenstein EB. The burdens of cancer therapy. Clinical and economic outcomes of chemotherapy-induced mucositis. Cancer. 2003;98(7):1531-9.

4. Vera-Llonch M, Oster G, Ford CM, Lu J, Sonis S. Oral mucositis and outcomes of allogeneic hematopoietic stem-cell transplantation in patients with hematologic malignancies. Support Care Cancer. 2007;15(5):491-6.

5. Balducci L, Exterrman M. Management of cancer in the older person: a practical approach. Oncologist. 2000;20:224-37.
6. Peterson DE, Cariello A. Mucosal damage: a major risk factor for severe complications after cytotoxic therapy. Sem Oncol. 2004;31:35-44.

7. Niscola P, Romani C, Cupelli L, Scaramucci L, Tendas A, Dentamaro T, et al. Mucositis in patients with hematologic malignancies: an overview. Haematologica. 2007;92:222-31.

8. Elting LS, Cooksley C, Chambers M, Cantor SB, Manzullo E, Rubenstein EB. The burdens of cancer therapy. Clinical and economic outcomes of chemotherapy-induced mucositis. Cancer. 2003;98(7):1531-9.

9. Sonis ST, Oster G, Fuchs H, Bellm L, Bradford WZ, Edelsberg J, et al. Oral mucositis and the clinical and economic outcomes of hematopoietic stem-cell transplantation. J Clin Oncol. 2001;19(8):2201-5.

10. Tomlinson D, Isitt J, Barron R, Doyle J, Judd P, Gassas A, et al. Determining the understandability and acceptability of an oral mucositis daily questionnaire. J Pediatr Oncol Nurs. 2008;25(2):107-11.

11. Wong D, Baker C. Pain in children: Comparison of assessment scales. Pediatr Nurs. 1998;14:9-17.

12. Ip WY, Epstein JB, Lee V, Yuen HL, Li R, Thompson DR, et al. Oral mucositis in paediatric patients after chemotherapy for cancer. Hong Kong Med J. 2014;20(Suppl 7):S4-8.

13. Witko-Sarsat V, Rieu $P$, Descamps-Latscha B, Lesavre $P$, Halbwachs-Mecarelli L. Neutrophils: molecules, functions and pathophysiological aspects. Lab Invest. 2000;80:61753.

14. Sonis ST, Etling LS, Keefe D, Peterson DE, Schubert M, Hauer-Jensen M, et al. Perspectives on cancer therapy-induced mucosal injury: pathogenesis, measurement, epidemiology, and consequences for patients. Cancer. 2004;100(9 Suppl):1995-2025. 